\title{
A Digitalized Farm Management Support System Using Wireless Sensor Network (WSN)
}

\author{
Yusuf Nura Muhammad, Issa Abdulmumeen Adekunle, Musa Maryam Abdullahi \\ Department of Mathematical Sciences, Abubakar Tafawa Balewa University, Bauchi, Nigeria \\ Email address: \\ ymnura@atbu.edu.ng (Y. N. Muhammad),aaissa@atbu.edu.ng (I. A. Adekunle),ammarya@atbu.edu.ng (M. M. Abdullahi)
}

\section{To cite this article:}

Yusuf Nura Muhammad, Issa Abdulmumeen Adekunle, Musa Maryam Abdullahi. A Digitalized Farm Management Support System Using Wireless Sensor Network (WSN). Internet of Things and Cloud Computing. Vol. 7, No. 4, 2019, pp. 95-109. doi: 10.11648/j.iotcc.20190704.12

Received: September 7, 2019; Accepted: October 8, 2019; Published: October 21, 2019

\begin{abstract}
The major challenge of irrigation farming is not having full control over the activities on the farmland and its unpredictable environment factors which in most cases, if not well managed, brings about low agricultural productivity. In this paper, as against the traditional manual control procedures which are time consuming, labor expensive, and most time led to taking bad key decisions concerning the three important environmental factors vis: temperature, humidity, and moisture of the farmland, we presents the design and implementation of farm management support system using WSN to sense and sent SMS about these three important farm field parameters to farmers using ATMEGA 328 controller. The system is designed to allow remote tracking of the parameters via an IoT cloud computing platform (ThingSpeak) and perform some Statistical analysis like temperature humidity variation and difference at a particular point in time on the sensed data using matlab. The developed system is scalable to incorporate the tracking of many other parameters and actions such as soil fertility, required soil nutrient, or trigger actions such as releasing water valve or activate alarming unit if certain parameter/s cross defined threshold. This research shows the importance of using wireless sensor network in precision farm field as the system solves the problem of continuous monitoring of data acquisition.
\end{abstract}

Keywords: IoT, ThingSpeak, WSN, Nodes, Sensors

\section{Introduction}

With the dwelling oil revenue Nigerian government had shifted it priorities to agriculture, because agriculture is considered to be the sector of the economy that has the capacity to give jobs to millions of people. So many programs such as Growth Enhancement Support Scheme (GES), Anchor Brower's Program (ABP), Rural Finance Institutions Building Program (RUFIN), The Nigeria Incentive-Base and Risk-Sharing for Agricultural Lending (NIRSAL) etc. are currently run by federal ministry of agriculture, CBN, States governments, in order to encourage the people to venture into agriculture. So far, about 78,000 farmers were trained and benefited in Kebbi state under the ABP Program alone and the program is extended to 14 other states. With these developmental plans of the government, modern agricultural technology obtainable in WSN technology can be utilize to improve the entire farm management efficiency and aid in decision making for better production yield in crop quality, assist in mitigating disaster and flooding risk using smart metering to monitor water flow, leakages and pollution extent in dams, river and other water sources, optimize precision in applying the right amount of farm inputs such as water, fertilizer, pesticides at the right location and at the right time, the technology can further help in keeping track of soil moisture, temperature, humidity and entire climate change on site and from meteorological network center.

\section{Theoretical Background}

In this section, comprehensive information about Wireless Sensor Network (WSN) and the existing applicable solutions with WSN have been described.

\subsection{Overviews of Wireless Sensor Network}

It has been pointed out that Wireless technologies have been under rapid revolution recently. Variety of wireless technologies being developed range from simple and short range (IrDA) that uses infrared light, point-to-point communications, to another short range wireless personal area 
network (WPAN) for point-to multi-point communications, such as Bluetooth (which uses line of sight) and ZigBee, to mid-range, multi-hop wireless local area network (WLAN), to long-distance cellular phone systems, such as GSM/GPRS and CDMA. Among the advantages of Wireless sensors when compares to wired sensors, is that the reliability of signal transmission is enhanced because wires are not used in the transmission process [13]. An insight to the general architecture of a wireless sensor node as shown in figure 1. It can be seen from the diagram that a wireless sensor node is made up of four major components, the sensing unit, the processing unit, the power unit and transceiver unit. The sensing unit is responsible for converting the measured physical quantities such as humidity, pressure, temperature, fluid level, rate of flow, position, moisture, pollution extent and concentration, etc. into a voltage signal and subsequently, passes it through digitalization process in order to produce digital output for processing. While in the other hand, the processing unit is equipped with a microcontroller which controls all of the functions of the sensor node and manages the communication protocols to carry out specific tasks. To make communication between the WSN node and network possible, a wireless transceiver unit is provided for that function. And lastly, the power unit, being the most critical component of a sensor node, supplies the necessary energy to bring life into all of these mentioned units [8]. Besides these major components, a sensor node may be customized to also include application depended components like power generator, location finding system and mobilizer. Power generators, such as solar cells, may be utilized to provide redundancy to the main power unit for prolonging the sensor node lifetime.

Hardware

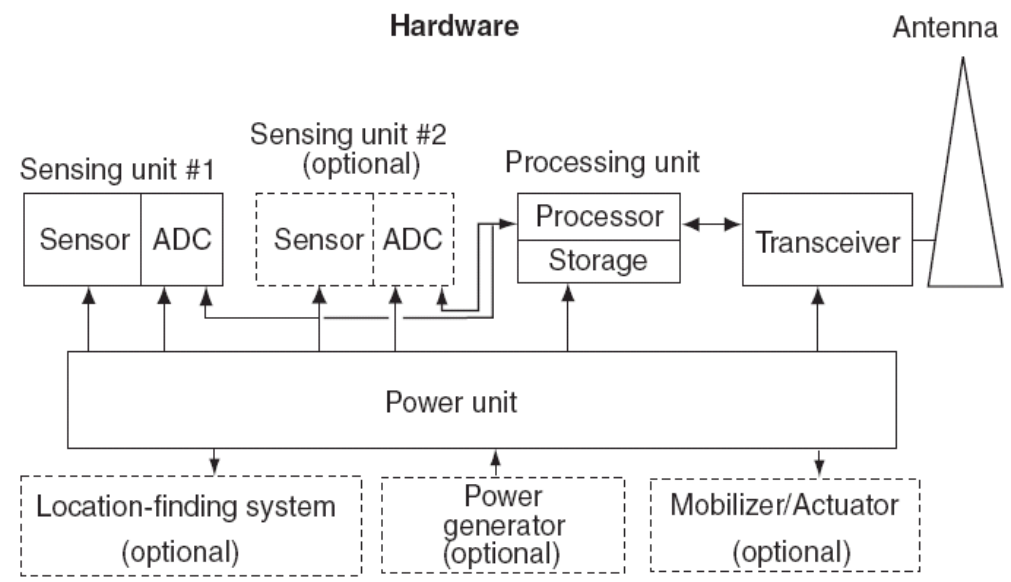

Figure 1. Wireless Sensor Node Architecture.

When two or more wireless sensor nodes are joined or connected together, they formed a network called wireless sensor network (WSN), the SNs are mostly composed of four subsystems comprising of power supply unit, sensing unit, processing unit, and radio communication unit. It was pointed out that wireless sensor networks (WSNs) are rigorously utilized in many applications area in order to optimize procedure or solve unresolved problems due to their capabilities [10]. One of its most widely employed ability is sensing. WSN has the ability to sense a surrounding physical phenomenon such as temperature, pressure, moisture, fluid level, contamination, etc.; and send the captured parameter as an input data for processing and onward transmission as processed information to the gateway via neighboring nodes/multiple-hops for wireless radio communication to the destination host. WSN has also been described as consisting large amounts of wireless sensor nodes, which are compact, light-weighted, and battery-powered devices that can be used in virtually any environment [2]. It is these special characteristics that made the sensor nodes to be usually deployed near the targets of interest in order to do close-range sensing. The data collected will undergo in-network processes and then return to the user who is usually located in a remote site. Most of the time, wireless sensor nodes are located in extreme environments, where are too hostile for maintenance.
Therefore, WSNs consist of multiple sensor nodes (SNs) for sensing and measurements collection, a gateway that is responsible for aggregating, processing, and analyzing the measured data, and software/MAC protocol which manages the allocation and operation of SN resources in a controlled manner. These capabilities make the technology as an ideal solution for applications in control system, systems monitoring and surveillance, smart homes, smart cities and many more. Some of the most critical areas of concern in wireless sensor networks deployment in any application area is that of coverage, connectivity and desire for high energy efficiency since sensor nodes are ordinarily energized by batteries that are characterized with low power and limited stored energy, which cannot be conveniently replaced or recharged. The main friction or challenges suffered by WSN technology are Resources limitation. Lifetime inadequacy, Diversity in Design, Quality of Service (QoS) requirements [12].

\subsection{Existing Applicable Solutions with WSN}

Applications of WSN can be classified into five categories:

1. Environmental monitoring

2. Precision agriculture

3. Machine and process control 
4. Building and facility automation and

5. Traceability systems.

With respect to precision agriculture, WSN Wireless sensors have been used to assist in

i. Spatial data collection

ii. Precision irrigation

iii. Variable-rate technology and

iv. Supplying data to farmers. [13]

See Wang, et.al, 2005 for a comprehensive list of researches and solutions provided by WSN in precision agriculture.

In Spatial data collection; a mobile field data acquisition system was developed to acquire data for crop control and spatial-variability analysis. The system is made up of a data collection sub system, a manager sub system and data acquisition and control systems farm machines. The system succeeded in carrying out local field survey and gathered data of soil water availability, soil compaction, soil fertility, biomass yield, leaf area index, leaf temperature, leaf chlorophyll content, plant water status, local climate data, insect-disease-weed infestation, grain yield, etc. [6]. In another effort, a silage yield mapping system, which comprises a GPS, load cells, a moisture sensor and a Bluetooth wireless communication module was designed. The moisture sensor and the Bluetooth transmitter were installed on a chopper. Signals from the moisture sensor was transmitted via a Bluetooth receiver on a host $\mathrm{PC}$ at a data rate of $115 \mathrm{kbps}$ and was used to correct the yield data [9]. Similarly, a joined effort with a private company led to a wireless, infrared thermometer system for in-field data collection. The system consisted of infrared sensors, programmable logic controllers and low power radio transceivers to collect data in the field and transmit it to a remote receiver outside the field [12].

Precision irrigation; However, in applying WSN in precision irrigation cumulate to a designed and evaluated an automatic and distributed irrigation system that remotely controlled, a 1500 ha irrigated area in Spain. The farm land area was divided into seven sub-regions with a total of 1850 hydrants (an upright water pipe) installed. Each sub-region was monitored and controlled by a control sector. The seven control sectors communicated to each other and with a central control through a WLAN network. Field tests showed $30-60 \%$ saving in water usage [4].

For Variable-rate technology in precision agriculture, WSN technology has been applied through the development of an automated fertilizer applicator for tree crops. The system comprised an input module for GPS and real-time sensor data acquisition, a decision module for calculating the optimal quantity and spread pattern for a fertilizer, and an output module to regulate the fertilizer application rate. Data communications among the modules were established using a Bluetooth network [3].

Supplying data to farmers; in optimizing data gathering procedure, the developed web server helped as information bank to famers with respect to pest and disease infestation and weather forecasts. Farmers can download the information directly via Internet and use them for operation scheduling [7]. USDA conducted a research in Mississippi to develop a high-speed wireless networking system to help farmers download aerial images via WLAN to their PCs, laptops or PDAs. The images were mainly used for precision farming applications [5]. WSN was designed for potato farming with the aim of tracking the potato field humidity. The WSN node is consisting of intelligent humidity sensors, microcontroller and low power radio transceivers to collect data in the field and transmit it to a remote receiver outside the field [8]. A framework of a wireless sensor network system was proposed to monitor various parameters on agricultural farmland in order to improve the yield. The sensors on the field are to sense environmental parameters which are transmitted to the base station and stored in the database [15]. In the work of Sherine, et.al, "precision farming solution in Egypt using wireless sensor network technology", the sensing modalities adopted is made up all in one sensor module which contain sensors for temperature, humidity, light intensity, soil $\mathrm{pH}$, and soil moisture [11]. The propose irrigation management system for controlling irrigation schedules of container grown crops by Khan, et.al, 2013, is based on temperature, air humidity and soil moisture. There are two basic units in the system design, the hardware unit and the software unit. The hardware unit comprises a wasp mote agriculture sensor boards that is specially designed for handling different agricultural activities. It can support up to 14 different sensors at the same time and switches to different operational modes for prolonging batteries lifetime, a wasp-mote gateway that acts as a bridge between sensor nodes and server [14]. Getha, and Priya, presented a smart irrigation control using WSNs, GSM and Android phone. It supports android phone applications for plant diseases monitoring and uses fuzzy logic techniques implemented with Arduino UNO board to check plant leaf infection and abnormality from its image, the image processing techniques is achieved with MATLAB [16]. A system that deals with efficient irrigation water management using distributed in-field sensor-based irrigation systems was developed to support site-specific irrigation management, the system allows farmers to maximize their productivity while saving water [18]. Also, in Mobile Network Supported Wireless Sensor Network Services, a Wireless Sensor network architecture that focused on the integration of WSNs into existing networks infrastructures that can interconnect independent WSN is used to provides sensor based and enhanced services such as data aggregation and actuators control services to remote users; primarily mobile network users [17].

In view of the endless applications of WSN in the literature, we present in this write up, a digitalized system for monitoring and controlling farmland activities and generate data in a digitized form as against the existing traditional wired form which consumed more time and less efficient.

\section{Materials and Method}

In this section, various aspects of the proposed digitalized farm management support system are presented.

\subsection{Flowchart of the System Plan}

The diagram below gives the flowchart of research plan. 


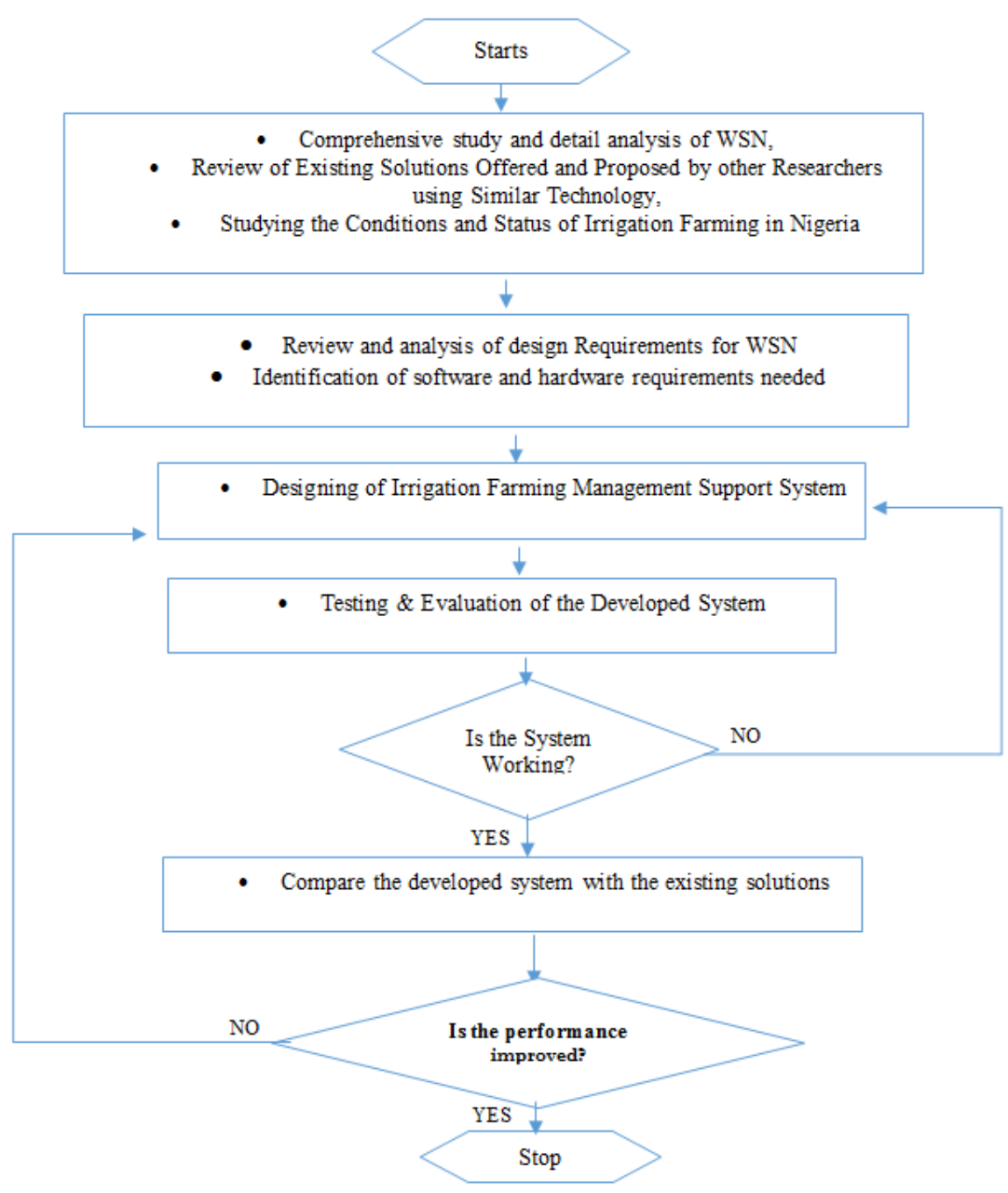

Figure 2. Flow Chart of Research Plan.

\subsection{Design Requirements}

The following hardware, software and other supporting tools were used to achieve the aim and objectives earlier outlined; Arduino Uno board (Figure 3), the Sensors (Temperature Sensor, Humidity Sensor, Moisture Sensor), Wi-Fi-module,
GSM module, ATMEGA 328 controller (figure 4), GSM phone, ThingSpeak, (an Internet of Things (IoT) Platform), power source, jumper cables, LED display and laptop.

i.) Arduino Uno board:

Figure 3 present the sample look of the Arduino Uno Board.

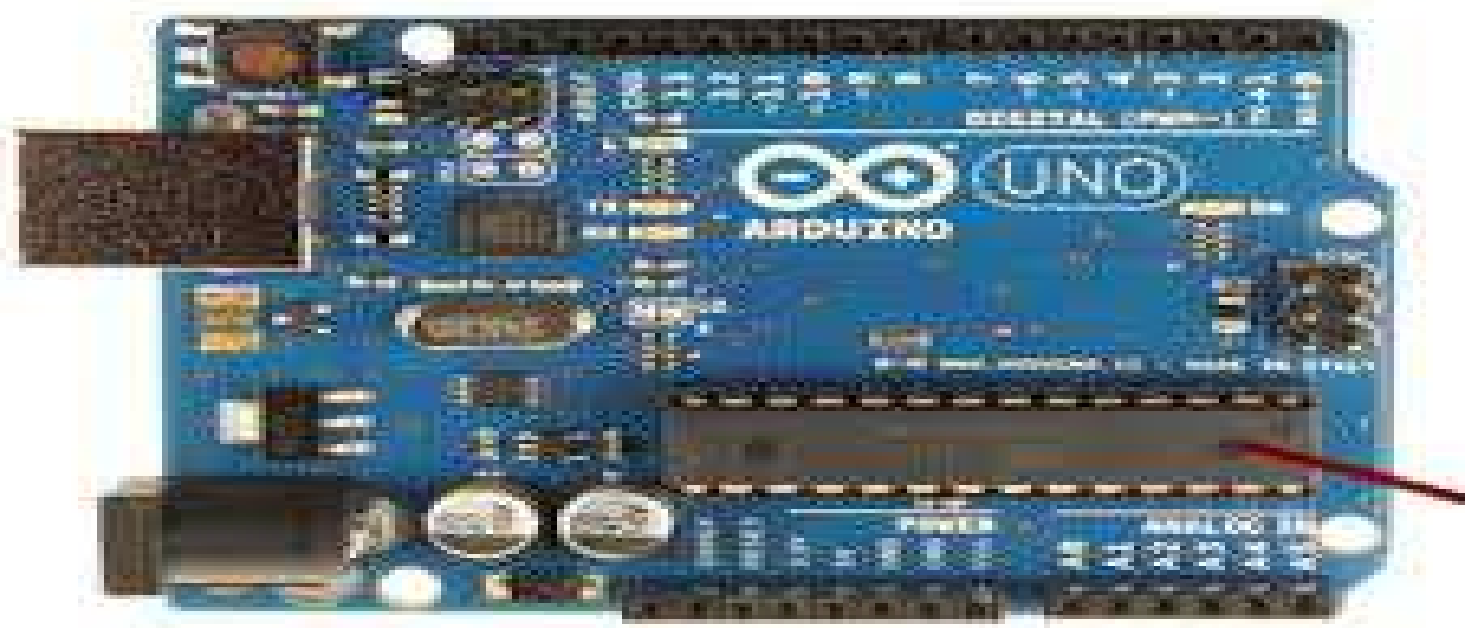

Figure 3. Arduino Board. 
ii.) ATMEGA 328 controller

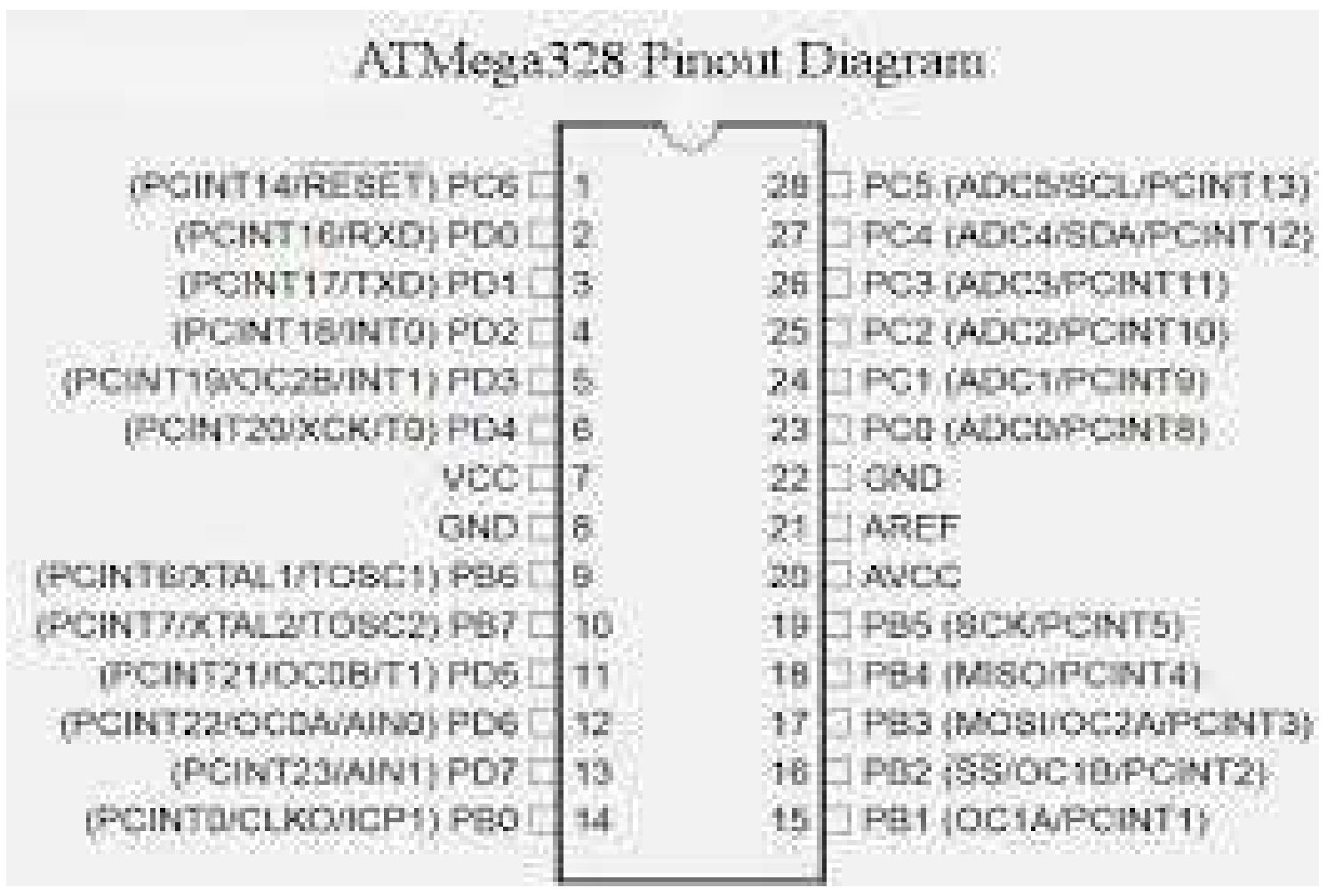

Figure 4. AMEGA 328 Microcontroller pinout.

iii.) The Sensors

Sensor nodes contained sensors for temperature, humidity and soil moisture. These sensing modalities exists in market in variations of hardware, for example, one sensor board such as MTS400 contains sensors.

a. The Temperature Sensor

LM35 IC is used for sensing the temperature. It is an integrated circuit sensor that can be used to measure temperature with an electrical output proportional to the temperature (in ${ }^{\circ} \mathrm{C}$ ). The sensor circuitry is sealed and not subject to oxidation, etc. it has a converter that accurately provides linear and directly proportional output signal in mill volts over the temperature range of $0^{\circ} \mathrm{C}$ to $155^{\circ} \mathrm{C}$.

b. The Humidity Sensor

The humidity sensor HIH4000, manufactured by Honeywell is used for sensing the humidity. It delivers instrumentation quality RH (Relative Humidity) sensing performance in a low cost, solderable SIP (Single Inline Package). The voltage is converted to the digital form by the ADC and then sent as input to the microcontroller which reads the data.

\subsection{Programming Used Include: Arduino IDE, C, and Node. Js}

The software includes the reading of various measurements from sensors, converting analog to digital values, displaying in the LCD module and sending the data to PC via HTTP port for monitoring the farm parameters from the webpage. Arduino Integrated Development Environment (Arduino IDE): Arduino IDE is used to program the microcontroller. The PC have the programming software Arduino c, this provides programming capabilities topology map, data export capability, mote programming and a command interface to sensor networks, web browser. A ThingSpeak. An open source Internet of Things (IoT) platform and API that lets you receive and mine sensor data in the cloud and develop IoT applications. It is also an IoT analytics platform service that allows you to aggregate, visualize and analyze real time data streams in the cloud using HTTP OSI application layer protocol over the Internet. ThingSpeak provides instant visualizations of data sensed and posted by the sensor device from the field. It also allow you to do the statistical analysis and visualization your data in MATLAB. It is used for prototyping and proof of concept of the developed system "The farm management support system".

\subsection{Architecture of the Designed System}

The system is made up four different layers, as shown in Figure 5.

i.) The Physical layer.

The Physical layer is comprised of the sensor-nodes deployed in the farm field which is made up of Soil Moisture Sensors Module, Temperature Sensor Module, Humidity Sensor Module, respectively. The Sensor-nodes are 
responsible for sensing different physical parameters in the farm field. The sensor-node sends the sensed information to the mote nodes through a physical serial interface. The mote nodes will be configured an adaptive energy efficient MAC protocol. The sensed 6LoWPAN packets will then be forwarded to the Edge Router acting as the Sink or Gateway.
Dual Stack protocol will be configured on the Edge Router, which gives it ability to communicate with the mote the IP Network. The Edge Router performs packet transformation from the 6LoWPAN packets to the IPv6 packets which is then sent to the IP-Network (Internet).

\begin{tabular}{|c|}
\hline Application layer \\
\hline Network Layer \\
\hline MAC \\
\hline Physical Layer \\
\hline
\end{tabular}

Figure 5. System Architecture.

ii.) Application Layer Interface Configuration diagrams in figures 6, 7, 8 and 9 illustrate the configuration (ThingSpeak IoT Cloud Interface)

The Application layer was designed to read the sensed parameters. Thus, the UDP payload will appear to the CMC with IP capability depicting the moisture, humidity, and temperature readings respectively. ThingSpeak IoT platform API and interface was used for integration of the system with IPv4-based Control Management center. The of the application layer/user interface of the farm management support system. It shows how a channel is created and setup to collect the farm parameters of interest and how the channel is linked with the generated API keys to view the captured data privately or publicly. The summary of the configuration is given in table 1 .

Table 1. API Configuration Summary.

\begin{tabular}{|c|c|c|c|c|c|}
\hline \multirow{2}{*}{ Channel Name/Description } & \multirow{2}{*}{ Channel ID } & \multicolumn{2}{|l|}{ API Keys } & \multirow{2}{*}{ Channel Stings } & \\
\hline & & Private & Sharing & & \\
\hline \multirow{3}{*}{ Farm Parameters } & \multirow{3}{*}{395139} & \multirow{3}{*}{ ZGTDOTQZVDXJ4 } & \multirow{3}{*}{ XWIVTJMIT39YJG } & Field 1 & Humidity \\
\hline & & & & Field 2 & Temperature \\
\hline & & & & Field 3 & Moisture \\
\hline
\end{tabular}

\section{$\square_{\checkmark}$ ThingSpeakru Channels - Apps Community Support - How to Buy Account - Sign Out}

\section{My Channels}

\section{New Channel}

\begin{tabular}{|l|l|l|}
\hline Name & Created & Updated At \\
\hline a farm parameters & $2018-01-02$ & 2018-01-02 11:02 \\
\hline
\end{tabular}

\section{Help}

Collect data in a Thingspeak channel from a device, from another channel, or from the web.

Click New Channel to create a new ThingSpeak channel.

Click on the column headers of the table to sort by the entries in that column.

Learn to create channels, explore and transform data.

Learn more about ThingSpeak Channels.

\section{Examples}

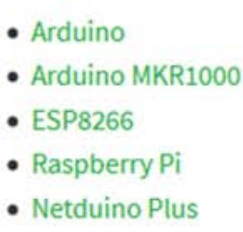

- Arduino

- Arduino MKR1000

- ESP8266

- Netduino Plus 


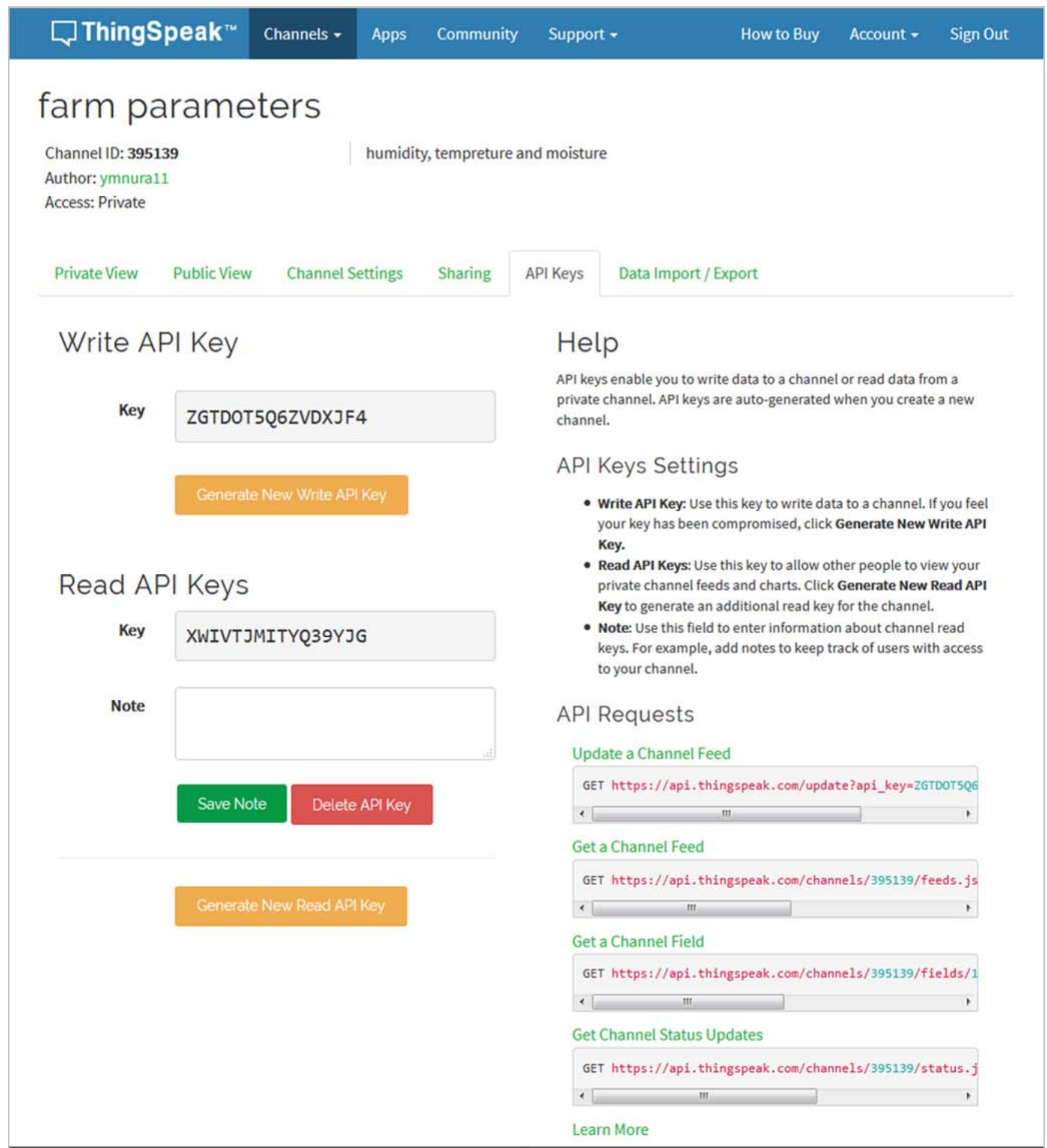




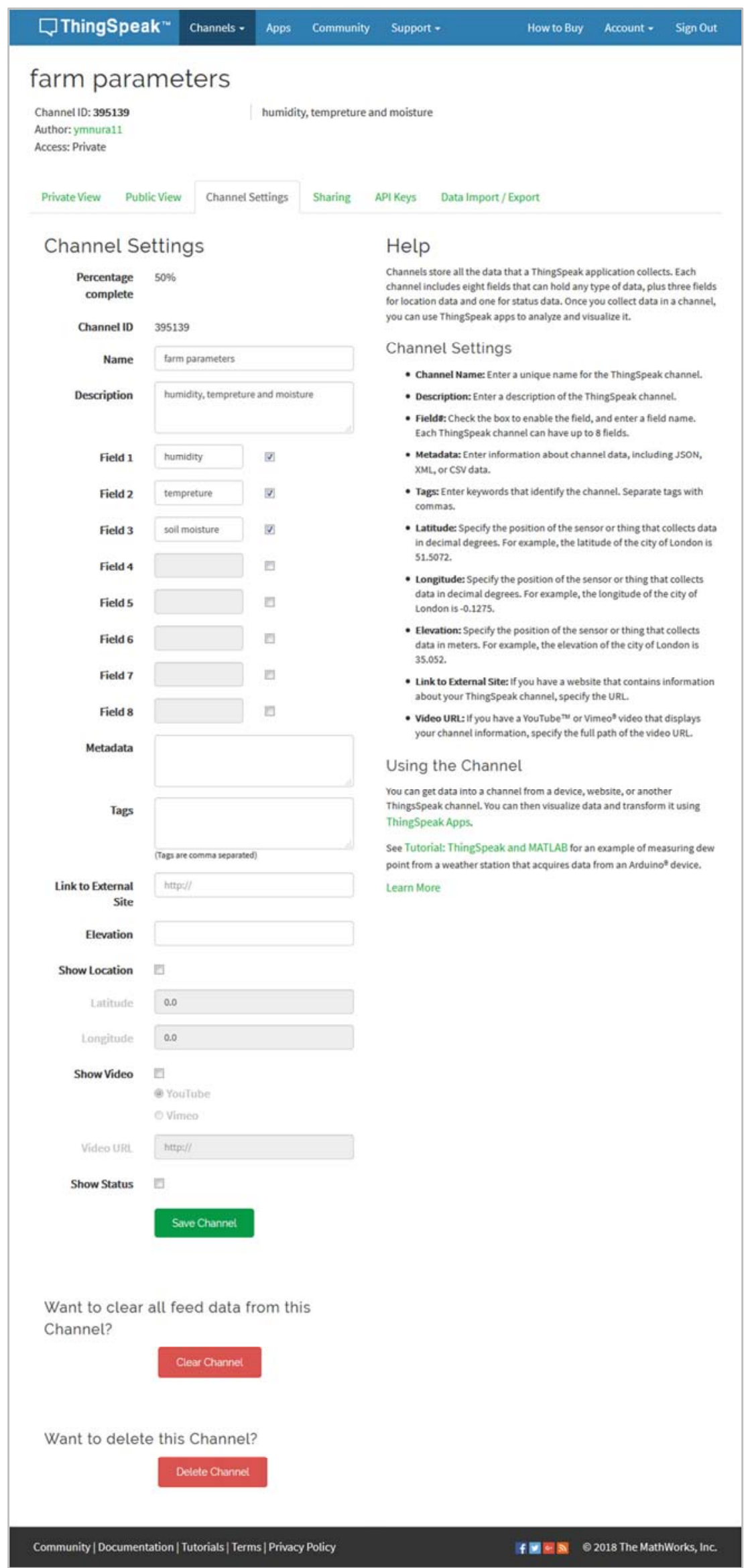

Figure 8. Channel Settings Interface. 


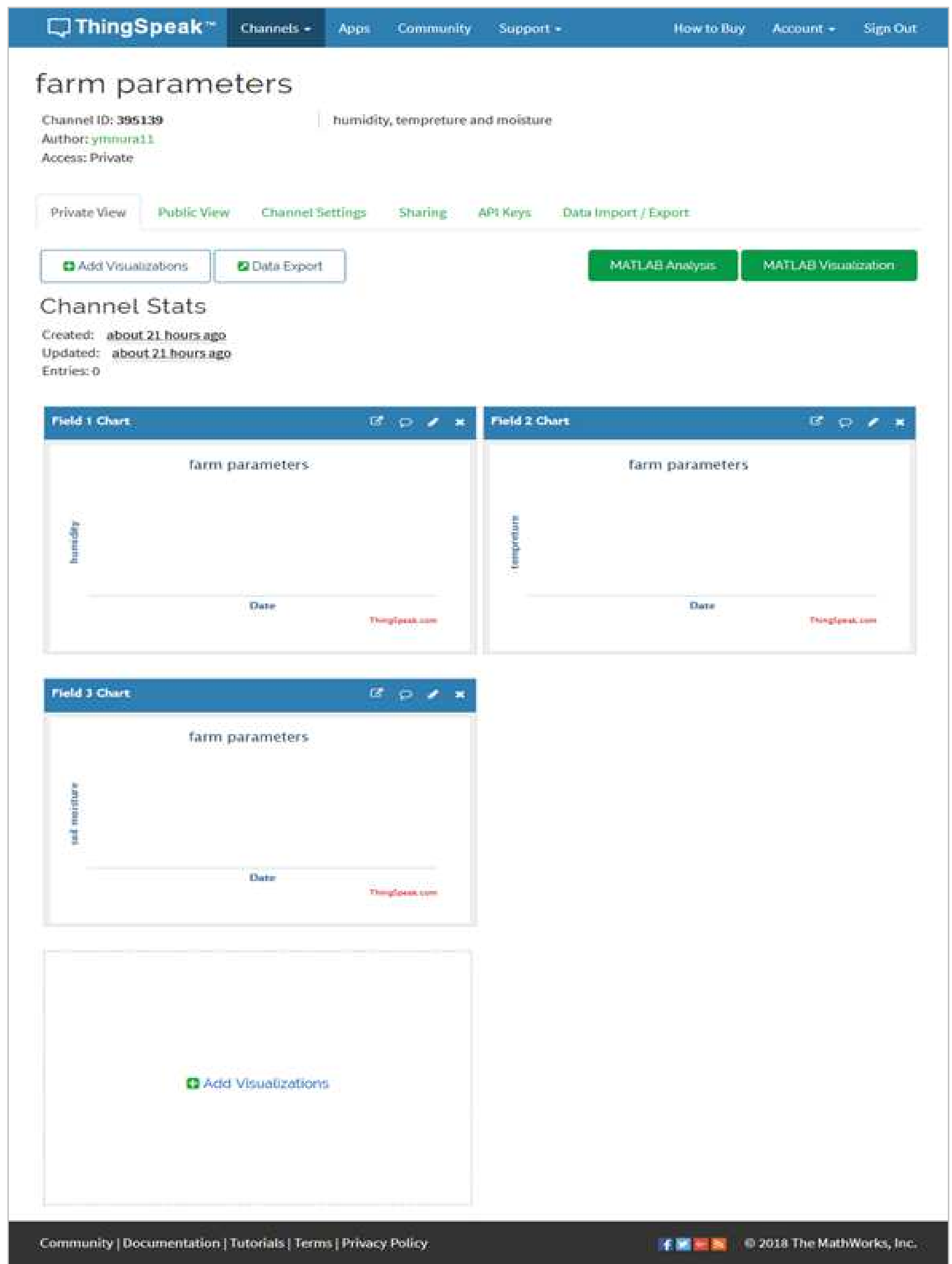

Figure 9. Parameters Interface. 


\subsection{The Conceptual System Design Layout}
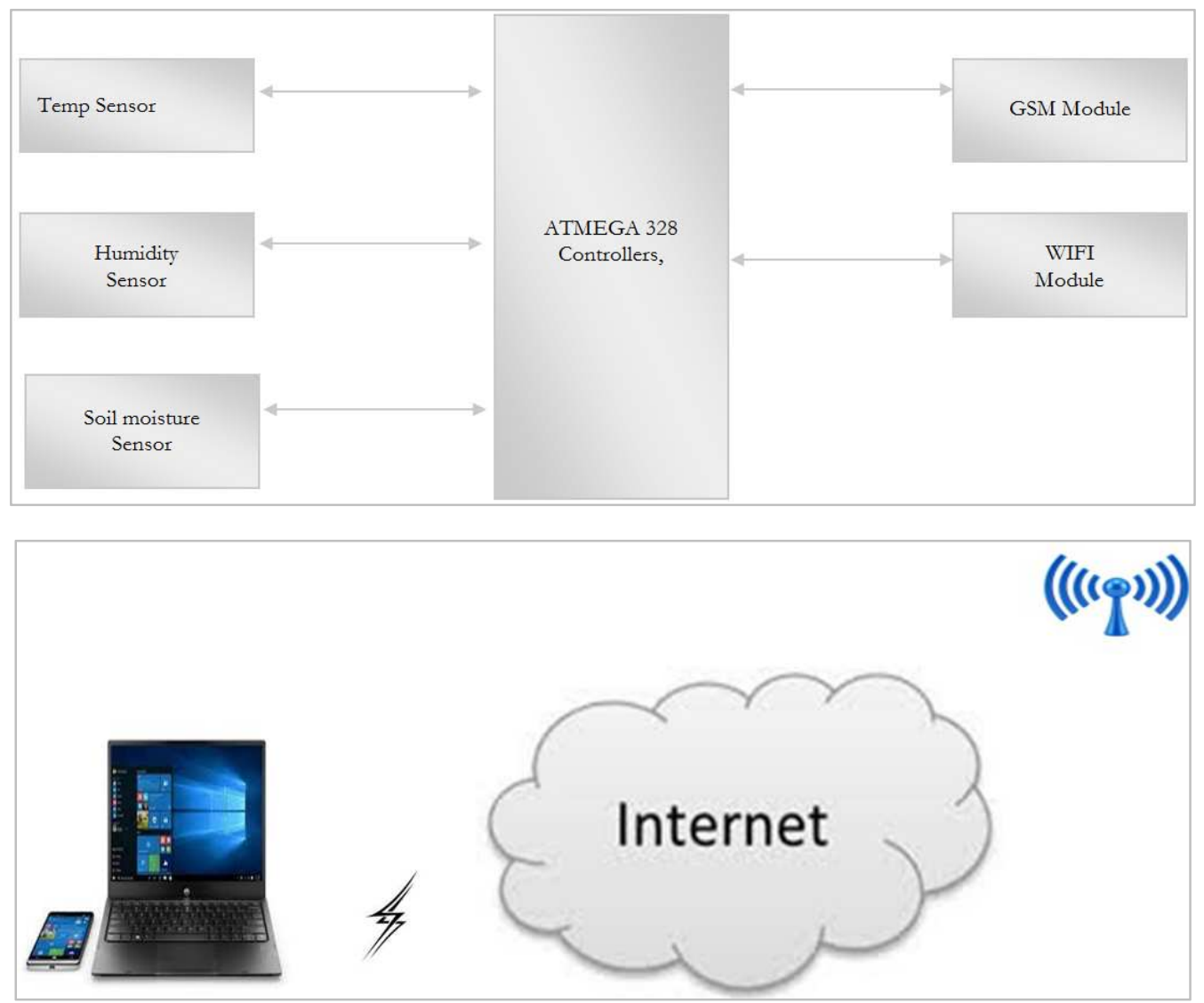

Figure 10. Conceptual System Design layout.

\section{Results and Discussion}

This passage shows the results of implementations for the proposed Digitalized Farm Management System. The variability of the resulting data has been analyzed using Matlab. The screenshots in figure 11 show the picture of the finished and final working farm management system. The
LCD is used to display messages that can be view onsite. For example, one of the display a message shows the value of

Humidity, Temperature and Soil moisture at some point in time.

In the event that the farm manager is not onsite or any authorized person with necessary credentials can track the parameters from anywhere remotely via a web browser as show in figure 12

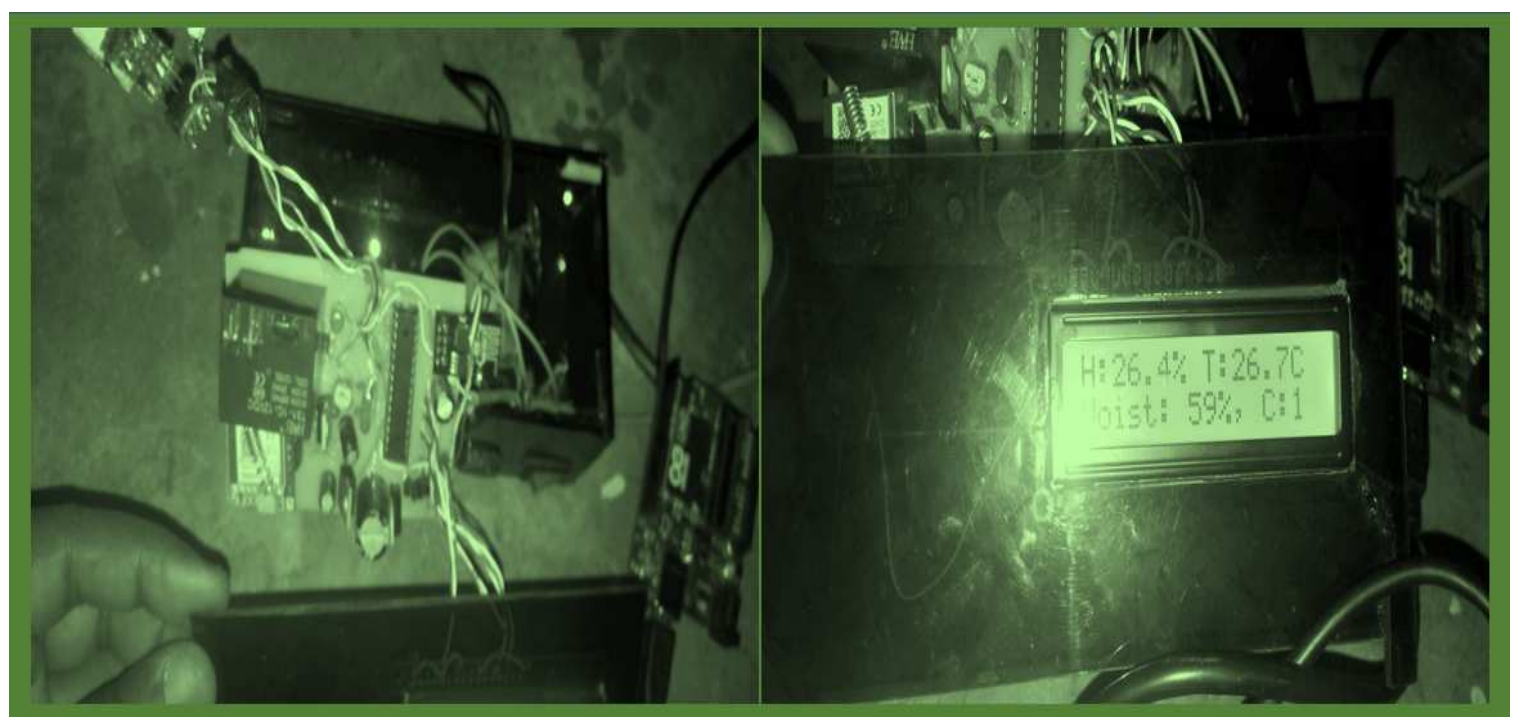

Figure 11. The working system. 


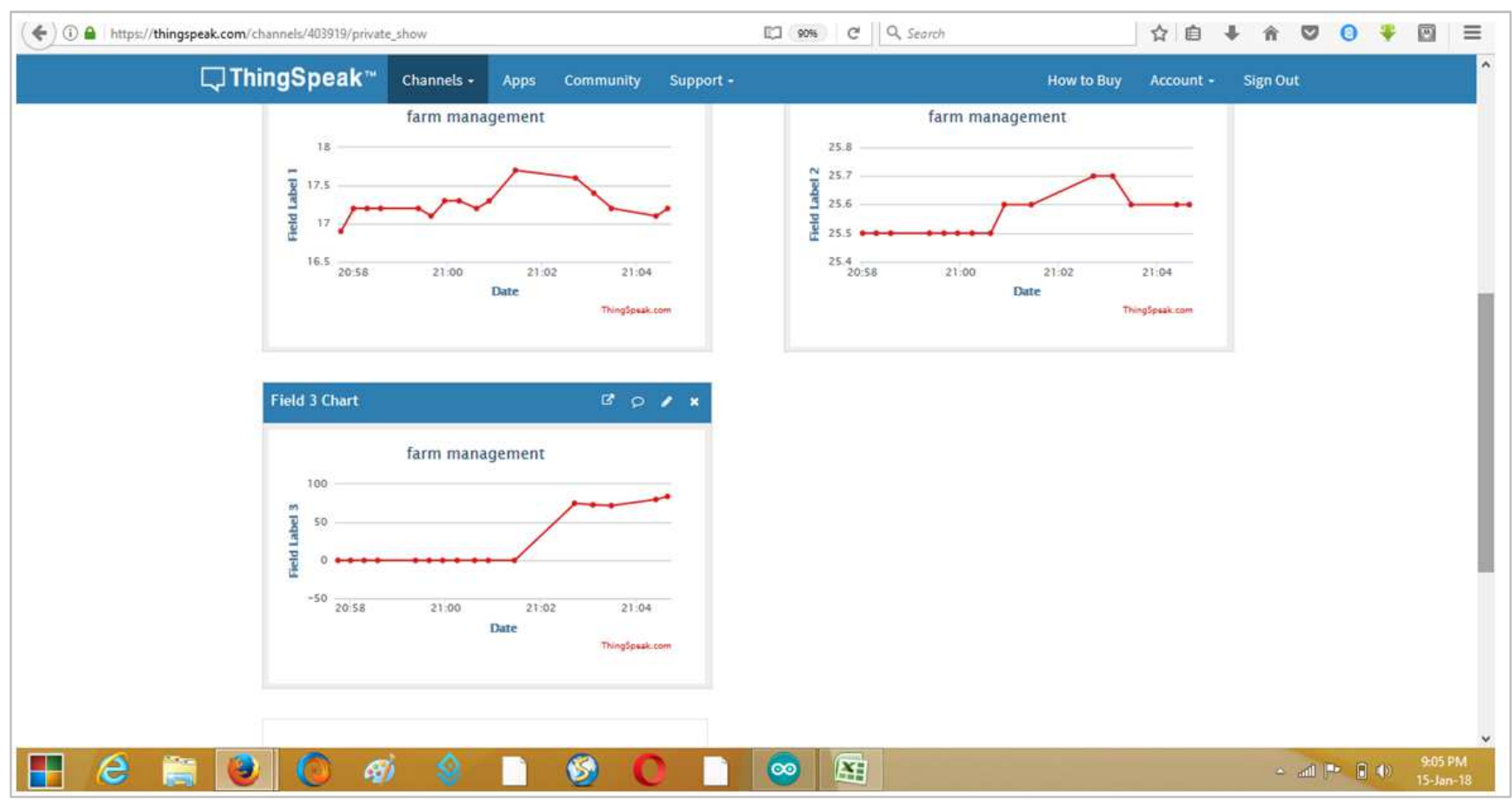

Figure 12. Line graph of Sample Data viewed from ThingSpeak interface on $15^{\text {th }}$ January 2018.

The ThingSpeak platform is so rich and flexible in such a way that one has the option to view the captured data in three (3) different file format viz: XML file, Java script object notation and one can choose to extract the data in csv (excel) format and present it in a tabular form. A Sample csv data obtained from the sensors is presented below and further statistical analyze in line graph as shown in figure 12. Erleaved modular multiplication method which requires 257 clock cycles. This modular multiplier need 1470 slice LUTs and 514 LUT-FF pairs with a total time of 2.09 ps.

Table 2. CSV formant of the Extracted data from the system.

\begin{tabular}{|c|c|c|c|c|}
\hline Entry id & Created at & Humidity & Temperature & Moisture \\
\hline 21 & 2017-12-28 13:19:59 UTC & 16.5 & 31.1 & 0 \\
\hline 22 & 2017-12-28 13:20:15 UTC & 16.3 & 31.1 & 0 \\
\hline 23 & 2017-12-28 13:20:32 UTC & 16.4 & 31.1 & 0 \\
\hline 24 & 2017-12-28 13:21:06 UTC & 15.5 & 31 & 1 \\
\hline 25 & 2017-12-28 13:21:27 UTC & 16.2 & 30.9 & 0 \\
\hline 26 & 2017-12-28 13:21:43 UTC & 17 & 30.9 & 0 \\
\hline 27 & 2017-12-28 13:22:00 UTC & 16.7 & 31 & 0 \\
\hline 28 & 2018-01-05 19:46:56 UTC & 20.2 & 27.2 & 0 \\
\hline 29 & 2018-01-05 19:47:12 UTC & 19.3 & 25.7 & 0 \\
\hline 30 & 2018-01-05 19:47:30 UTC & 19.9 & 25.7 & 0 \\
\hline 31 & 2018-01-05 19:47:46 UTC & 19.6 & 25.6 & 0 \\
\hline 32 & 2018-01-05 19:48:03 UTC & 19.4 & 25.5 & 0 \\
\hline 33 & 2018-01-05 19:48:35 UTC & 19.5 & 25.4 & 0 \\
\hline 34 & 2018-01-05 19:48:51 UTC & 19.4 & 25.4 & 0 \\
\hline 35 & 2018-01-05 19:49:10 UTC & 19.2 & 25.3 & 0 \\
\hline 36 & 2018-01-05 19:49:29 UTC & 19.3 & 25.3 & 0 \\
\hline 37 & 2018-01-05 19:49:47 UTC & 18.9 & 25.2 & 0 \\
\hline 38 & 2018-01-05 19:50:05 UTC & 18.4 & 25.1 & 0 \\
\hline 39 & 2018-01-05 19:50:20 UTC & 18.3 & 25.1 & 0 \\
\hline 40 & 2018-01-05 19:50:37 UTC & 18.3 & 25 & 0 \\
\hline 41 & 2018-01-05 19:50:54 UTC & 18.6 & 25 & 0 \\
\hline 42 & 2018-01-05 19:51:16 UTC & 18.6 & 24.9 & 0 \\
\hline 43 & 2018-01-05 19:51:33 UTC & 18.6 & 24.9 & 0 \\
\hline 44 & 2018-01-05 19:51:50 UTC & 18.6 & 24.9 & 0 \\
\hline 45 & 2018-01-05 19:52:06 UTC & 18.5 & 24.8 & 0 \\
\hline 46 & 2018-01-05 19:52:23 UTC & 18.4 & 24.8 & 0 \\
\hline 47 & 2018-01-05 19:52:45 UTC & 18.2 & 24.8 & 0 \\
\hline
\end{tabular}




\begin{tabular}{lllll}
\hline Entry id & Created at & Humidity & Temperature & Moisture \\
\hline 48 & $2018-01-05$ 19:53:02 UTC & 18.6 & 24.7 & 0 \\
49 & $2018-01-05$ 19:53:19 UTC & 18.6 & 24.7 & 0 \\
50 & $2018-01-05$ 19:53:35 UTC & 18.4 & 24.7 & 0 \\
51 & $2018-01-05$ 19:53:53 UTC & 18.3 & 24.6 & 96 \\
52 & $2018-01-05$ 19:54:11 UTC & 18.4 & 24.6 & 97 \\
53 & $2018-01-0519: 54: 29$ UTC & 18.2 & 24.6 & 94 \\
54 & $2018-01-05$ 19:54:46 UTC & 18 & 24.5 & 91 \\
55 & $2018-01-05$ 19:55:10 UTC & 17.9 & 24.5 & 86 \\
56 & $2018-01-0519: 55: 28$ UTC & 17.9 & 24.5 & 80 \\
57 & $2018-01-0519: 55: 45$ UTC & 18 & 24.4 & 78 \\
58 & $2018-01-0519: 56: 03$ UTC & 17.8 & 24.4 & 76 \\
59 & $2018-01-0519: 56: 21$ UTC & 17.8 & 24.3 & 73 \\
60 & $2018-01-0519: 56: 38$ UTC & 17.8 & 24.3 & 66 \\
\hline
\end{tabular}

Graphical display of the variability of generated data for the farm parameters is as given in figures 13, 14, 15 and 16 below. jointly, figures 14,15 , and 16 give separate display of While figure 13 presents the variability of the farm parameters variability in each of the farm parameters.

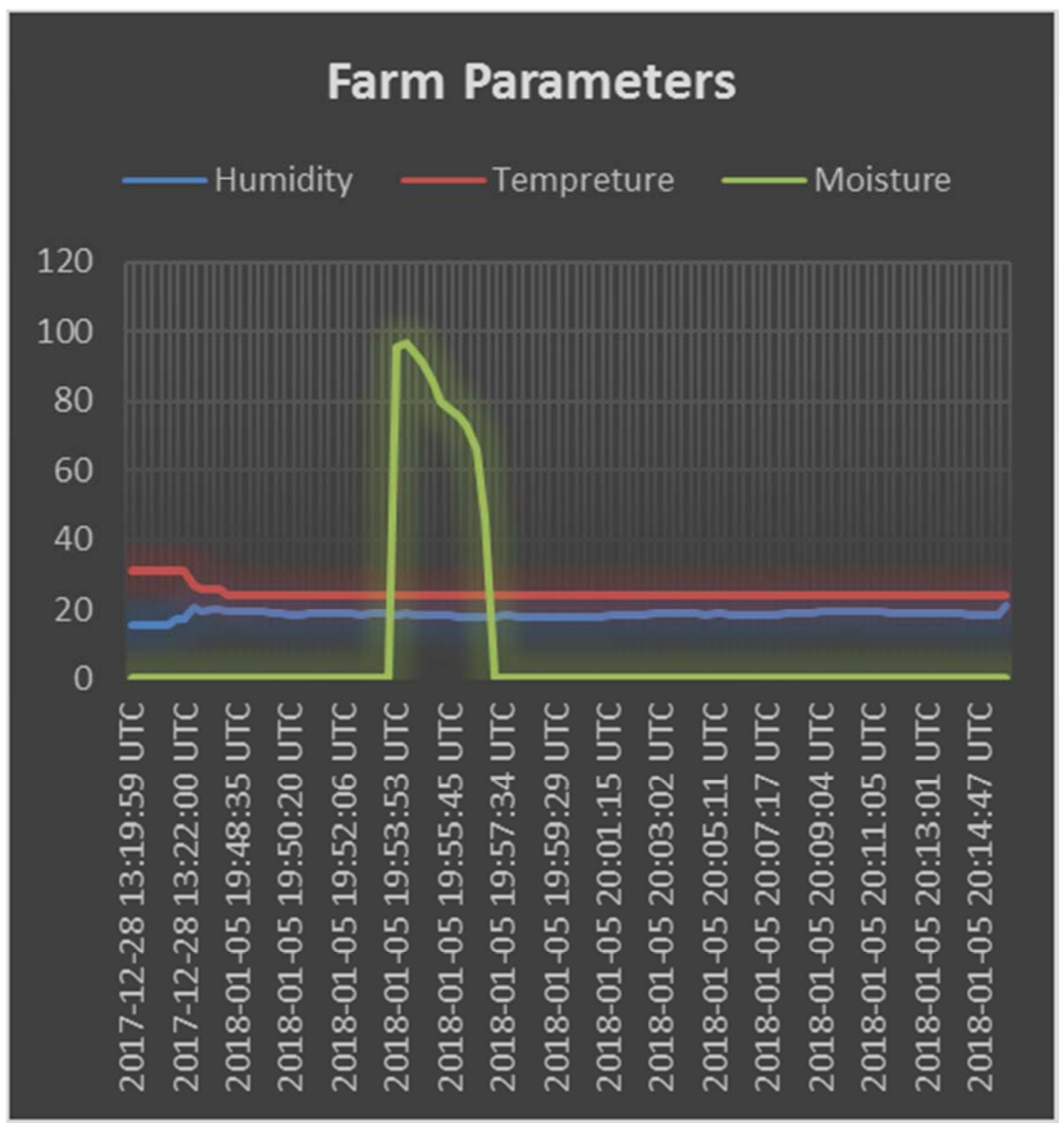

Figure 13. Joint display of variability of the farm parameters. 


\section{Humidity}

22

21

20

19

18

17

16

15

14

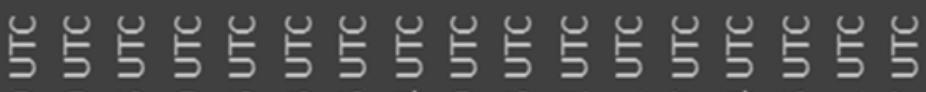
గ్ధ

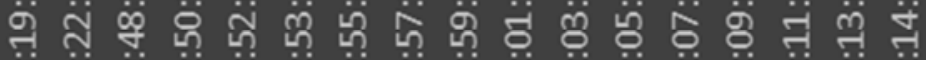

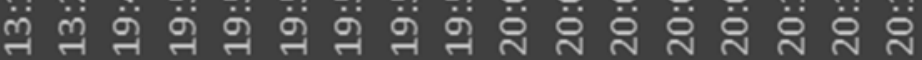
疋

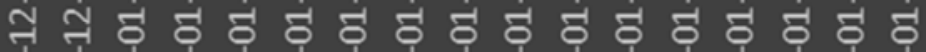

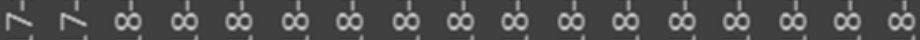

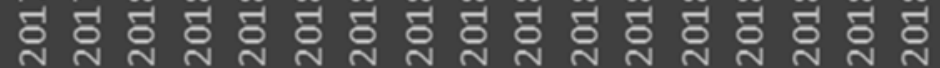

Figure 14. Display of variability of the farm Humidity.

\section{Tempreture}




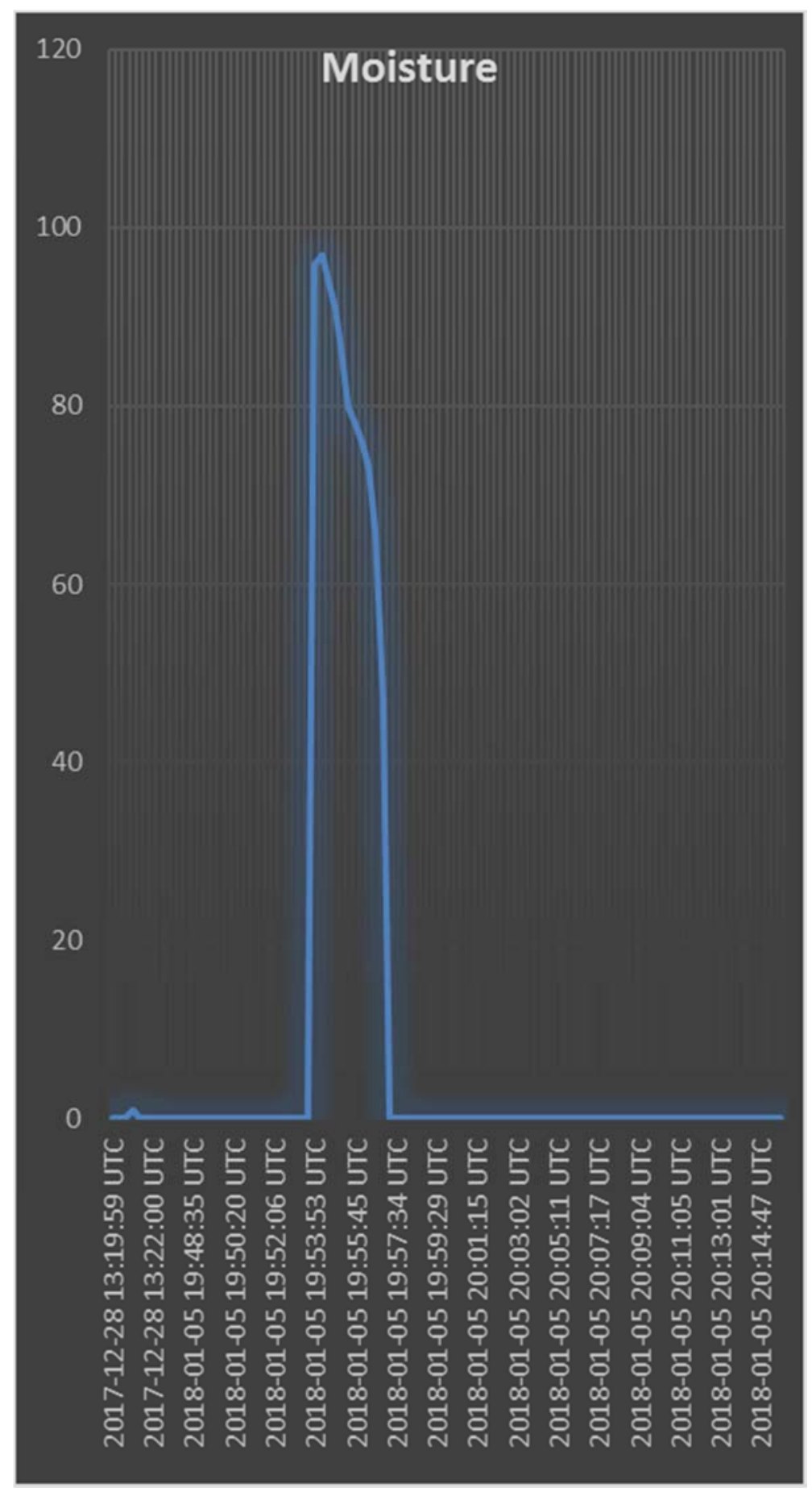

Figure 16. Display of variability of the farm Moisture.

\section{Conclusion and Recommendations}

\subsection{Conclusion}

This paper shows the importance of using wireless sensor network in precision farming field. The importance of optimized and efficient management support system in agriculture cannot be overemphasized especially the dry season farming (irrigation system) that takes decisions over crops with respect to soil water level and environmental parameters very seriously. While the traditional manual procedures are time consuming, labor expensive that usually leads to bad judgment in taking key decisions, in the other hand, the designed system will enhance farming techniques and help to solve or mitigate the identified contemporary problems and consequently improve the crops yield and reduce wastage of resources.

\subsection{Recommendations}

Presently, wireless sensor-based systems comprising of microprocessors with communication capabilities can connect to an infinite network of THINGS to monitor, track and affect 
changes and actions on behalf of humans. This concept can be applied to any field, including agriculture as demonstrated in this paper. Wireless sensors and sensor networks have entered farms and from the look of things they will have a bright future, I therefore, recommend our farmers to embrace it and enjoy the benefits it provides. But before, usage we need to validate and verify these models for use in different environments, equally important, I recommend cost benefit of WSNs analysis to farmers. There is a need to asses, evaluate and quantify profit or the return on investment that a farmer could be attracted to, and to be able to scale those benefits for small medium and large producers, with respect to WSN scale.

\section{References}

[1] KB. S. Fakayode, O. O. (2008). An Economic Survey of Rural Infrastructures and Agricultural Productivity Profiles in Nigeria,. European Journal of Social Sciences, 7 (2), 158-171.

[2] C. Cheng, C. T. (March 2011). A Delay-Aware Data Collection Network Structure for Wireless Sensor Networks.. IEEE Sensors Journal, Vol. 11, (No. 3).

[3] Cugati, S. M. (June 2003). Automation concepts for the variable rate fertilizer applicator for tree farming. The Proceedings of the 4th European Conference in Precision Agriculture, (pp. 14-19). Berlin, Germany,.

[4] Damas, M. P. (2001). fieldbus for integrated management of extensive areas of irrigated land. Microprocessors Microsyst, $25,177-184$.

[5] Flores, A. (2003, Frost and Sullivan,.). Speeding up data delivery for precision agriculture. Agric. Res. Mag. Retrieved from The United State Department of Agriculture (USDA: http://www.freescale.com, http://www.mindbranch.

[6] Gomide, R. I. (2001). An automatic data acquisition and control mobile laboratory network for crop production systems data management and spatial variability studies in the Brazilian center-west region. The American Society of Agriculture Engineers, (ASAE Paper) (No.: 01-1046.).

[7] Jensen, A. B. (2000). a web-based system for personalized decision support in crop management. Comput. Elect. Agric.
[8] K. Shinghal, A. N. (2010). Intelligent Humidity Sensor for Wireless Sensor Network for potato farming. International Journal of Engineering Science and Technology, Vol. 2 (8), 3955-3963.

[9] Lee, W. B. (2002). Silage yield monitoring system. The American Society of Agriculture Engineers, ASAE Paper (No.: 02-1165.).

[10] M. Abdelaal, O. T. (December 2-4, 2013). An Efficient and Adaptive Data Compression Technique for Energy Conservation in Wireless Sensor Networks. IEEE conference on wireless sensor (ICWiSe2013),. kuching, Sarawak: IEEE.

[11] M. Sherine, A. E.-k.-B. (2013). Precision farming solution in Egypt using the wireless sensor network technology. Egyptian Informatics Journal (2013), 14, 221-233.

[12] Mahan, J. W. (2004). Upchurch, Design and Construction of a Wireless Infrared Thermometry System.. The USDA Annual Report. Project Number: 6208-21000-012-03.

[13] N. Wang, N. M. ((2005) 501-14.). Wireless sensors in agriculture and food industry-Recent development and future perspective; Computers and Electronics in Agriculture. available online at www.science direct.com.

[14] R. Khan, I. A. (2013). Wireless Sensor Network Based Irrigation Management System for Container Grown Crops in Pakistan. World Applied Sciences Journal, 24 (8), 1111-1118.

[15] S. Adebayo, A. A. (October, 2015). Increasing Agricultural Productivity in Nigeria Using Wireless Sensor Network (WSN). African Journal of Computing \& ICT, Vol 8. (No. 3 Issue 2).

[16] S. Getha, R. P. (2016). Smart Irrigation Control Using WSNs, GSM and Android phone,. Asian journal of information technology, 15 (19), 3780-3786.

[17] Srdjan Krco, V. T. (2007). Mobile Network Supported Wireless Sensor Network Services. IEEE.

[18] Y. J. Kim, R. G. (July 2008.). Network, Remote Sensing and Control of an Irrigation System Using a Distributed Wireless Sensor;. IEEE Transactions On Instrumentation and Measurement, 57. 